\title{
Cervical Cancer Vaccination: A New Hope
}

\author{
Maruf Siddiqui \\ Dr. Maruf Siddiqui, Asstt. Professor, Department of Obstetrics \& Gynaecology \\ Anwer Khan Modern Medical College.Dhancondi, Dhaka
}

\begin{abstract}
Cervical cancer remains a threat which is second only to breast cancer in overall disease burden for women throughout the world. Organized screening with Pap cytology has made substantial reductions in cervical cancer morbidity and mortality in recent years. Establishment of genital human papillomaviruses (HPVs) as the etiological agent and invention of HPV vaccines now provide a great opportunity which could be useful in saving millions of lives in future. Initial Phase I, II and early phase III trials have shown promising results of this vaccine. This review article therefore is aimed to explore different aspects of this vaccine as well as to develop awareness among health professionals of different disciplines.
\end{abstract}

Key Words : Cervical Cancer, Vaccination, Review

\section{Introduction}

Cervical cancer remains a critical public health problem that is second only to breast cancer in overall disease burden for women throughout the world ${ }^{1}$. Universal deployment of organized or opportunistic screening with Pap cytology in high and middle income countries has been the primary reason for the substantial reductions in cervical cancer morbidity and mortality during the last 50 years. However, in many low income countries Pap cytology screening is yet to be effectively implemented or has failed to reduce cervical cancer rates to an appreciable extent ${ }^{2}$. Screening appears to benefit only a small fraction of women although a much larger percentage endures the inconvenience of the Pap test in order to avoid cervical cancer.

A wealth of epidemiological and molecular evidence has led to the conclusion that virtually all cases of cervical cancer and its precursor intraepithelial lesions are a result of infection with one or other of a subset of genital human papillomaviruses (HPVs) suggesting that prevention of infection by prophylactic vaccination would be an effective anti-cancer strategy.

To date, more than 120 genotypes of Human Papillomavirus (HPV) are known, but only high risk oncogen genotypes could induce a cancer. HPV 16 and 18 are implied in nearly $70 \%$ of cervical cancer around the world ${ }^{3}$. The condylomata (condyloma acuminatum), or genital warts, induced by HPV 6 and 11 are frequent among the young and difficult to manage. The extent and burden of HPV infection are considerable, as is the psychological and emotional impact of the diseases associated with it. A prophylactic vaccine to protect against the precancerous and cancerous lesions associated with HPV should save lives, reduce expensive diagnostic and therapeutic interventions, and have substantial individual and collective benefits ${ }^{3}$.

\section{Mechanism of action of vaccines}

Based on the induction of neutralizing antibodies by non infectious Virus Like Particles (VLP) of L1 capside protein, prophylactic HPV vaccines have consistently induced high titter of neutralizing antibodies with minimal side effects and induce more than $90 \%$ protection from persistent HPV $16-$ 18 infection and HPV 16 and 18 associated highgrade Cervical Intraepithelial Neoplasia (CIN) in proof of concept efficacy trials. HPV 16-18 vaccination will prevent HPV 16-18 incident infection, and subsequently decrease in $90 \%$ the frequency of abnormal Pap attributable to these 
types and in about $50 \%$ overall abnormal Pap. HPV vaccination will reduce the number of women who require colposcopy, biopsy and cervical treatment for precancerous cervical lesions ${ }^{1}$. The level of protection from death due to cervical cancer could exceed 95\%. HPV vaccination targeting young female adolescents, aged 11 to 16 years, with a catch-up of those aged 17-25 years, would be a strategy to be addressed. Cervical cancer screening strategies that will be cost-effective for the proper surveillance of women protected by HPV vaccination ${ }^{1}$.

The papillomaviruses cannot be grown in large amounts in culture in vitro, but the ability to generate HPV virus like particles (VLPs) by the synthesis and self-assembly in vitro of the major virus capsid protein L1 provides for a potentially effective sub unit vaccine. HPV L1 VLP vaccines are immunogenic and have a good safety profile. Published data from proof of principle trials and preliminary reports from large Phase III efficacy trials suggest strongly that they will protect against persistent HPV infection and cervical intra epithelial neoplasia ${ }^{4}$. However, the duration of protection provided by these vaccines is not known, the antibody responses induced are probably HPV type specific and immunization should occur pre-exposure to the virus. Second generation vaccines could include an early antigen for protection post-exposure and alternative delivery systems may be needed for the developing world ${ }^{4}$.

\section{Bivalent (Cervarix) and Quadrivalent (Gardasil) Vaccines}

Although some persistent HPV infections progress to cervical cancer, host immunity is generally able to clear most HPV infections providing an opportunity for cervical cancer prevention through vaccination. Candidate prophylactic vaccines based on papillomavirus L1 virus-like particles (VLPs) are currently on human clinical trials: one targeting cervical cancer with a bivalent VLP L1 vaccine (Cervarix: marketed by GlaxoSmithKline Biologicals, Rixensart, Belgium.) containing the two genotypes most frequently involved in cervical cancer (type 16 and 18) and the other, protecting against warts as well as cervical cancer, with a quadrivalent HPV VLP L1 vaccine (Gardasil: marketed by MERCK \& Co., USA \& Canada) containing genotypes $6,11,16$ and 18 . The first clinical trials revealed the satisfactory tolerance and excellent immunogenicity of these vaccines inducing high serum antibody titers with minimal side effects ${ }^{5}$. After more than three years, both clinical trials on women 15 to 25 years old have shown that vaccines are able to type specifically protect against nearly $90 \%$ of infection and all cervical intra-epithelial neoplasia ${ }^{5}$.

The two new HPV vaccines (Gardasil-quadrivalent and Cervarix-bivalent) are both effective against HPV types 16 and 18, which are responsible for $70 \%$ of cervical cancers, and the quadrivalent vaccine is also effective against HPV 6 and 11, responsible for genital warts ${ }^{6}$. Their efficacy is $100 \%$ if they are administered before exposure to HPV $16,18^{6}$. The proven duration of protection against high-grade cervical lesions is currently 5 years, given in a 3 -dose series $(0$ month, 1 month and 6 month or 0 month, 2 month and 6 month) and the need for boosters is unknown. Cervical cancer screening programs must continue, as only $70 \%$ of the 15 high-risk HPV types are targeted ${ }^{6}$. The best age for primary vaccination appears to be 11-12 or 14 years, before the outset of sexual activity $^{6}$.

Include Pain at the injection site (about 8 people in $10)$, Redness or swelling at the injection site (about 1 person in 4), Mild fever $\left(100^{\circ} \mathrm{F}\right.$ ) (about 1 person in 10), Itching at the injection site (about 1 person in 30 ) and Moderate fever $\left(102{ }^{\circ} \mathrm{F}\right)$ (about 1 person in 65) ${ }^{7}$. However, these symptoms do not last long and life-threatening allergic reactions from vaccines are very rare ${ }^{7}$.

\section{Initial reports of Trials of Vaccine}

A follow-up, multicentre, double-blind, randomised, placebo-controlled trial reported in 2004 on 393 patients, the researchers found that more than $98 \%$ seropositivity was maintained for HPV-16/18 antibodies during the extended follow-up phase ${ }^{8}$. Significant vaccine efficacy was noted against HPV-16 and HPV-18 endpoints: incident infection, 
96.9\% (95\% CI 81.3-99.9); persistent infection: 6 month definition, 94.3 (63.2-99.9); 12 month definition, $100 \%$ (33.6-100). In a combined analysis of the initial efficacy and extended follow-up studies, vaccine efficacy of $100 \%$ (42.4100 ) against cervical intraepithelial neoplasia (CIN) lesions associated with vaccine types ${ }^{8}$. The concluding remarks are: up to 4.5 years, the HPV$16 / 18$ L1 virus-like particle AS 04 vaccine is highly immunogenic and safe, and induces a high degree of protection against HPV-16/18 infection and associated cervical lesions. There is also evidence of cross protection ${ }^{8}$.

In another randomized, double-blind, controlled trial $^{9}$ to assess the efficacy, safety, and immunogenicity of a bivalent HPV-16/18 L1 viruslike particle vaccine for the prevention of incident and persistent infection with these two virus types, associated cervical cytological abnormalities, and precancerous lesions; Researchers randomized 1113 women between 15-25 years of age to receive three doses of either the vaccine formulated with AS 04 adjuvant or placebo on a 0 month, 1 month, and 6 month schedule in North America and Brazil. Women were assessed for HPV infection by cervical cytology and selfobtained cervicovaginal samples for up to 27 months, and for vaccine safety and immunogenicity. In the according-to-protocol analyses, vaccine efficacy was $91.6 \%$ (95\% CI 64.5-98.0) against incident infection and $100 \%$ against persistent infection (47.0-100) with HPV-16/18. In the intention-to-treat analyses, vaccine efficacy was 95.1\% (63.5-99.3) against persistent cervical infection with HPV-16/18 and 92.9\% (70.0-98.3) against cytological abnormalities associated with HPV-16/18 infection. The vaccine was generally safe, well tolerated, and highly immunogenic ${ }^{9}$. So, they concluded that the bivalent HPV vaccine was efficacious in prevention of incident and persistent cervical infections with HPV-16 and HPV-18, and associated cytological abnormalities and lesions ${ }^{9}$.

Another group of researchers from New Zealand conducted a randomized, double-blind, phase II or III trials in $>19,000$ women aged $15-25$ years, in which the HPV 16/18 vaccine (Cervarix) showed high efficacy in preventing CIN $2+$ associated with
HPV 16/18. Cross-protection against new incident or 6-month persistent HPV 45 or HPV 31 infection was also evident. In a bridging study, in adolescent girls aged 10-14 years, Cervarix induced twice the already high antibody titres as it did in young women (aged 15-25 years). The vaccine was generally well tolerated and injection-site reactions were the most common vaccine-related adverse events reported ${ }^{10}$.

To gauge the potential impact on disease burden, Rambout L et al performed a systematic review of the evidence from randomized controlled trials ${ }^{11}$. They conducted a systematic search of the literature to identify all randomized controlled trials of prophylactic HPV vaccination. Reports in 5 electronic databases covering 1950 to June 2007 (MEDLINE, MEDLINE in process, EMBASE, the Cochrane Central Registry of Controlled Trials and the Cochrane Library), bibliographies of all included studies and of narrative reviews (20062007), clinical trial registries, Google Scholar, public health announcements, selected conference proceedings (2004-2007) and manufacturers' information on unpublished data or ongoing trials were screened against predefined eligibility criteria by 2 independent reviewers. The primary outcome of interest was the frequency of high-grade cervical lesions (high-grade squamous intraepithelial lesion, or grade 2 or 3 cervical intraepithelial neoplasia). The secondary outcomes were persistent HPV infection, low-grade cervical lesions (low-grade squamous intraepithelial lesion or grade 1 cervical intraepithelial neoplasia), external genital lesions, adverse events and death. RESULTS: Of 456 screened reports, 9 were included in the review (6 were reports of randomized controlled trials and 3 were follow-up reports of initial trials). Findings from the meta-analysis showed that prophylactic HPV vaccination was associated with a reduction in the frequency of high-grade cervical lesions caused by vaccine-type HPV strains compared with control groups: Peto odds ratio 0.14 (95\% confidence interval $[\mathrm{CI}]$ 0.09-0.21) from combined perprotocol analyses, and 0.52 (95\% CI $0.43-0.63)$ from modified intention-to-treat analyses. Vaccination was also highly efficacious in preventing other HPV-related infection and disease 
outcomes, including persistent HPV infection, lowgrade lesions and genital warts ${ }^{11}$. The majority of adverse events were minor. INTERPRETATION: Among women aged 15-25 years not previously infected with vaccine-type HPV strains, prophylactic HPV vaccination appears to be highly efficacious in preventing HPV infection and precancerous cervical disease ${ }^{11}$.

\section{Vaccination Program, Strategies and Guidelines}

A national HPV vaccination program is due to begin in the UK in September 2008. Girls aged 12 to 13 years of age will be offered one of the vaccines, Cervarix, and it is hoped that it will offer them protection against over $70 \%$ of cervical cancer $^{12,13}$.

Different countries are now adopting and experimenting about the vaccination strategies. The American College of Obstetricians and Gynecologists (ACOG), The Royal College of Obstetricians and Gynaecologists (RCOG) and the Royal Australian and New Zealand College of Obstetricians \& Gynaecologists (RANZCOG) have already prepared their own guidelines. The Asia and Oceania Federation of Obstetrics and Gynaecology (AOFOG) recently issued some recommendations regarding this vaccine for this region.

\section{Recommendations of the Asia Oceania Federation of Obstetrics \& Gynaecology (AOFOG) on Cervical Vaccination ${ }^{14}$}

The AOFOG Advisory Recommendations was drafted at the Singapore Meeting on 2nd May 2008 , held in conjunction with the Inauguration of the AOFOG Cervical Cancer Awareness Month and endorsed by AOFOG Council on 27 June 2008, at the AOFOG Council Meeting held in Colombo, Sri Lanka. (Produced by AOFOG Gynaecological Oncology Committee). The recommendations are as follows:

Objective: The objective of the AOFOG Advisory Recommendations is to provide information on the use of HPV vaccine for the purpose of cervical cancer prevention and the need to continue with the current cervical screening programs.
General: The Human Papilloma Virus (high-risk genotypes) is a necessary causal factor of cervical cancers. The HPV Vaccine is a prophylactic vaccine. It protects against infection of HPV genotypes (HPV-16 and HPV-18) that account for about $70 \%$ of HPV-related cervical cancers. The HPV vaccine is not therapeutic. It does not treat existing cervical intraepithelial neoplasia (cervical pre-cancers).

Cervical Cancer Screening: Women who have been vaccinated with the HPV vaccine should continue with the cervical cancer screening as per the recommendations in their resident country.

Vaccination Target Group: The vaccine has been approved for use in females from 9 years old and the age licensure varies between countries. In some countries, it has also been approved for use in males. It should target females at the most convenient and optimal age (12-16 years old) for vaccination before their first sexual exposure.

Counseling before Vaccination: A full explanation of the role, action and usefulness of the vaccine should be provided for the woman or her parent/guardian where applicable, before vaccination. The explanation should typically include: the role of HPV in cervical carcinogenesis (in particular HPV-16 and HPV-18); trial results and expectations; immunological responses; safety and efficacy; as well as answer queries on issues, as highlighted in this document.

HPV Testing before Vaccination: Testing for HPV is currently not recommended before vaccination.

Vaccination of Sexually Active Women: Sexually active women and women with previous abnormal cervical cytology can receive the HPV vaccine. But the benefits may be limited to the protection against infection of HPV genotypes with which they have not been infected. Women who have been infected with vaccine HPV-type (serologically positive) and has cleared of the cervical infection (DNA negative), appears to have similar protective effects as those who are naïve to the same vaccine HPV-type. 


\section{Special Situations}

A) Women with Previous Cervical Intraepithelial Neoplasia (CIN): The vaccine can be given to patients with previous CIN, but the benefits may be limited to the protection against infection of HPV genotypes (and related CIN) with which they have not been infected. It must be emphasized that cervical cytology surveillance and corresponding management must continue.

B) Pregnancy and Lactating Women: The use of the vaccine in pregnancy is not recommended, although no teratogenic effect caused by the vaccine has been reported. There is no evidence to show that the HPV vaccine adversely affects fertility, pregnancy or infant outcome.

- Women who are planning to conceive are advised to defer vaccination until after delivery.

- Women who become pregnant before completion of vaccination are advised to postpone the remaining dose until after the pregnancy.

- Termination of pregnancy is not indicated for women who become inadvertently pregnant during the course of vaccination.

- Lactating women can receive the HPV vaccine and still continue breastfeeding because it is a vaccine without live viral DNA.

\section{C) Immunosuppressed Patients}

Immunosuppression is not a contraindication to vaccination. However, the immune response to the HPV vaccine may be less competent in these women compared with a healthy individual.

Contraindications and Precautions: The HPV vaccine is contraindicated for people with a history of hypersensitivity to any vaccine component. Vaccination of people with moderate or severe acute illnesses should be deferred until after the illness improves.

\section{Conclusion}

Both Cervarix and Gardasil recombinant vaccines have been effective in preventing persistent infection with targeted HPV types and in preventing cervical intraepithelial lesions, while
Gardasil has also been effective in preventing vulvar and vaginal neoplasia and genital warts. Both vaccines have been well tolerated, with the most common adverse effects occurring at the injection site.

Preliminary data indicate that the vaccination is effective in women who have previously eliminated naturally the virus. It has no therapeutic effects on existing lesions or in healthy virus carriers. Practical questions remain to be resolved. If the vaccination is left to individual initiative and vaccination coverage is insufficient, there will be no perceptible reduction in the frequency of cervical cancer. Vaccination policies will not be identical in poor countries, where the disease represents one of the leading causes of mortality among women, and in the rich countries, where screening programs have considerably reduced the frequency of this cancer. Current planning calls for the introduction of systematic vaccination of young girls aged 9-15 years, with progressive "catch-up" vaccination of the cohorts of young women aged 16-26 years. The progressive development of primary prevention strategies should coexist with secondary prevention with redesigned screening programs. The successful development of vaccination programs will require the support of public health authorities, the coordination of health workers from different areas and increased public awareness.

Nonetheless mathematical models and immunogenicity results indicate a possible benefit for individual vaccination of adults. This approach must still be assessed in the clinical trials underway. Because the vaccine does not protect against all types of HPV associated with cervical cancer, screening must be continued according to the conditions currently set. Vaccination and screening, which are complementary and synergistic, now constitute the new standards for prevention of this disease.

Phase I and II trials showed the safety of these vaccines and their capacity to produce very-high titers of antibodies (low or non-existent after natural infection). Phase III trials are ongoing to further evaluate vaccine efficacy. Issues remaining to be addressed include duration of protection, efficacy for prevention of cervical cancer, optimal age for vaccination, feasibility of application to the developing world, the ideal combination of HPV 
subtypes, and the most efficient combination of vaccination and cervical cancer screening.

Ongoing clinical studies are expected to provide further evidence of efficacy and will form the basis for licensing of candidate vaccines by the major pharmaceutical companies within 3-6 years. Although the future seems bright on the HPV vaccine front policy makers are strongly cautioned to avoid scaling back cervical cancer screening. It will possibly take many years before we can rationally develop cervical cancer screening strategies that will be cost-effective for the proper surveillance of women protected by HPV vaccination.

\section{References}

1. Monsonégo J. Cervical cancer prevention: the impact of HPV vaccination. J Gynecol Obstet Fertil. 2006 Mar; 34(3): 189-201.

2. Franco EL, Harper DM. Vaccination against human papillomavirus infection: a new paradigm in cervical cancer control. Vaccine. 2005 Mar 18; 23(17-18): 238894.

3. Monsonego J. [Prevention of cervical cancer (II): prophylactic HPV vaccination, current knowledge, practical procedures and new issues] Presse Med. 2007 Apr; 36(4 Pt 2): 640-66.

4. Stanley MA. Human papillomavirus vaccines. Rev Med Virol. 2006 May-Jun;16(3): 139-49.

5. Bégué $\mathrm{P}$, Henrion R, Blanc B, Girard M, Sancho-Garnier $\mathrm{H}$. Vaccination against human papillomavirus. Implementation and efficacy against cervical cancer control. Bull Acad Natl Med. 2007 Dec;191(9): 1805-16;

6. Frederick PJ, Huh WK; PATRICIA Study Group. Evaluation of the interim analysis from the PATRICIA study group: efficacy of a vaccine against HPV 16 and 18. Expert Rev Anticancer Ther. 2008 May; 8(5): 701-5

7. www.gardasil.com

8. Harper DM, Franco EL, Wheeler CM, Moscicki AB, Romanowski B, Roteli-Martins CM, Jenkins D, Schuind A, Costa Clemens SA, Dubin G; HPV Vaccine Study group. Sustained efficacy up to 4.5 years of a bivalent L1 virus-like particle vaccine against human papillomavirus types 16 and 18: follow-up from a randomised control trial. Lancet. 2006 Apr 15; 367(9518): 1247-55

9. Harper DM, Franco EL, Wheeler C, Ferris DG, Jenkins D, Schuind A, Zahaf T, Innis B, Naud P, De Carvalho NS, Roteli-Martins CM, Teixeira J, Blatter MM, Korn AP, Quint W, Dubin G; GlaxoSmithKline HPV Vaccine Study Group. Efficacy of a bivalent L1 virus-like particle vaccine in prevention of infection with human papillomavirus types 16 and 18 in young women: a randomised controlled trial. Lancet. 2004 Nov 13-19; 364(9447): 1757-65.

10. Keam SJ, Harper DM. Human papillomavirus types 16 and 18 vaccine (recombinant, AS04 adjuvanted, adsorbed) [Cervarix]. Drugs. 2008; 68(3): 359-72.

11. Rambout L, Hopkins L, Hutton B, Fergusson D. Prophylactic vaccination against human papillomavirus infection and disease in women: a systematic review of randomized controlled trials. CMAJ. 2007 Aug 28; 177(5): 469-79.

12. Paavonen J, Jenkins D, Bosch FX, Naud P, Salmerón J, Wheeler CM, Chow SN, Apter DL, Kitchener HC, Castellsague X, de Carvalho NS, Skinner SR, Harper DM, Hedrick JA, Jaisamrarn U, Limson GA, Dionne M, Quint W, Spiessens B, Peeters P, Struyf F, Wieting SL, Lehtinen MO, Dubin G; HPV PATRICIA study group. Efficacy of a prophylactic adjuvanted bivalent L1 viruslike-particle vaccine against infection with human papillomavirus types 16 and 18 in young women: an interim analysis of a phase III double-blind, randomised controlled trial. Lancet. 2007 Jun 30; 369(9580): 2161-70

13. Cadman L. The future of cervical cancer prevention: human papillomavirus vaccines. J Fam Health Care. 2008; 18(4): 131-2.

14. www.aofog.org

15. Bayas JM, Costas L, Muñoz A. Cervical cancer vaccination indications, efficacy, and side effects. Gynecol Oncol. 2008 Sep; 110(3 Suppl 2): S11-4.

16. Schmiedeskamp MR, Kockler DR. Human papillomavirus vaccines. Ann Pharmacother. 2006 JulAug; 40(7-8): 1344-52.

17. Brinkman JA, Caffrey AS, Muderspach LI, Roman LD, Kast WM.The impact of anti HPV vaccination on cervical cancer incidence and HPV induced cervical lesions: consequences for clinical management. Eur $J$ Gynaecol Oncol. 2005; 26(2): 129-42.

18. Gardasil ${ }^{\circledR}$ American Society of Health-System Pharmacists, Inc. Disclaimer AHFS $^{\circledR}$ Consumer Medication Information. (C) Copyright 2008. The American Society of Health-System Pharmacists, Inc., 7272 Wisconsin Avenue, Bethesda, Maryland.

19. Hantz S, Alain S, Denis F. Human papillomavirus prophylactic vaccines: stakes and perspectives. Gynecol Obstet Fertil. 2006 Jul-Aug; 34(7-8): 647-55

20. Williamson AL, Passmore JA, Rybicki EP. Strategies for the prevention of cervical cancer by human papillomavirus vaccination. Best Pract Res Clin Obstet Gynaecol. 2005 Aug; 19(4): 531-44.

21. Hantz S, Alain S, Denis F. Anti-papillomavirus vaccines and prevention of cervical cancer: progress and prospects. Presse Med. 2005 Jun 4; 34(10): 745-53 